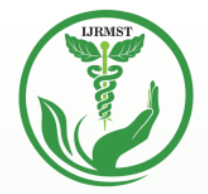

INTERNATIONAL JOURNAL OF RESEARCH IN MEDICAL SCIENCES \& TECHNOLOGY

e-ISSN:2455-5134; p-ISSN: 2455-9059

EMPLOYABILITY OF THE MACHINE LEARNING ALGORITHMS IN THE EARLY DETECTION AND DIAGNOSIS OF CARDIOVASCULAR DISEASES

\title{
Muskaan Juneja
}

Ramjas College, University of Delhi

Paper Received: 04 $4^{\text {th }}$ November, 2021; Paper Accepted: 20 $0^{\text {th }}$ November, 2021;

Paper Published: 24 ${ }^{\text {th }}$ December, 2021

DOI: http://doi.org/10.37648/ijrmst.vl li02.016

How to cite the article:

Muskaan Juneja, Employability of the Machine Learning Algorithms in the

Early Detection and Diagnosis of Cardiovascular Diseases, IJRMST, JulyDecember 2021, Vol 12, 181-189, DOI: http://doi.org/10.37648/ijrmst.v11i02.016

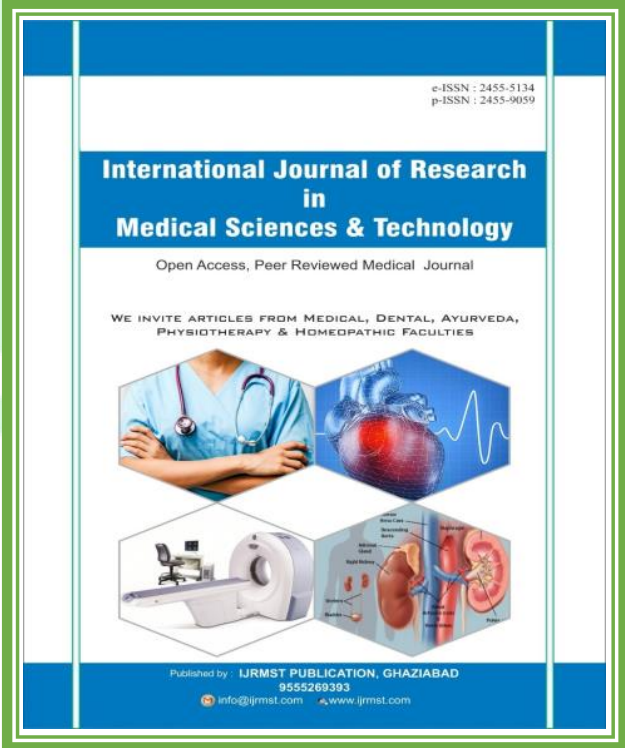




\section{VOLUME: 12, JULY-DECEMBER 2021}

\section{ABSTRACT}

AI (ML) is a fast-growing field these days. Use AI to separate information from a wide variety of sources. ML can tackle different issues dependent on complex informational collections. Because of intricacy, the handling of huge informational collections is more confused. Foreseeing coronary illness is the most challenging task in the clinical field. It can't be seen with the normal eye. It can show up quickly anyplace, whenever. Numerous ML calculations are more fit for dealing with different calculations. Working on these frameworks can work on the nature of clinical analysis options. They can observe designs concealed in a lot of information that will try not to involve conventional factual strategies for examination. In this research, we proposed an algorithm called Enhanced New Dynamic Data Processing (ENDDP) to anticipate the beginning phases of heart disease. The outcomes demonstrate the exhibition of the proposed framework.

\section{INTRODUCTION}

AI (ML) is mostly used in machine-based applications that can execute consequently. For everybody, the heart is an extremely sensitive and significant part. Human existence depends on the heartbeat. Many individuals experience the ill effects of heart disease because of old enough, propensities and different reasons. Breaking down a coronary attack in the beginning phases is truly irregular. Numerous sicknesses have been affirmed in the last stage because of the absence of precise data and comprehension of manifestations. The conclusion and coronary illness treatment are more confusing, particularly in emerging nations. Numerous passing's are happening because of the deficiency of specialists and other clinical-related gear [1]. Patients with coronary illness should go to lengths to lessen the danger of genuine coronary illness and further develop healthy hearts [2].

ML is fundamental to getting the best outcomes in any application. It is additionally more compelling in instructing and testing applications. It is a subset of (AI), one of the wide areas of AI that can replicate human capacities; ML is a significant part of AI. It is extremely valuable to foresee heart infections that have an area with ML. An ENDDP Algorithm has been acquainted with accomplishing more precise forecasts to conquer different difficulties in foreseeing coronary illness.

\section{DATA SET DESCRIPTION}

\section{Collection of Data and Pre-Processing}

The informational collection used the "coronary illness informational collection", a combination 


\section{VOLUME: 12, JULY-DECEMBER 2021}

of 4 distinct data sets; however, the UCI repository informational collection is used to collect data. The information base has 76 properties, yet distributed studies utilize a subset of 14 highlights to address [9]. Consequently, we utilized the handled UCI repository dataset on the Kaggle site for investigation. Table 1 records a full report of the 14 credits utilized in the proposed work.

\begin{tabular}{|c|c|c|}
\hline S1.No. & Attribute Description & Distinct Values of Attribute \\
\hline 1. & Age-represent the age of a person & Multiple values between $29 \& 71$ \\
\hline 2. & Sex-describe the gender of person ( 0 - Female, 1-Male) & 0,1 \\
\hline 3. & $\mathrm{CP}$ - represents the severity of chest pain patient is suffering. & $0,1,2,3$ \\
\hline 4. & RestB P-It represents the patients BP. & Multiple values between $94 \& 200$ \\
\hline 5. & Chol-It shows the cholesterol level of the patient. & Multiple values between $126 \& 564$ \\
\hline 6. & FBS-It represents the fasting blood sugar in the patient. & 0,1 \\
\hline 7. & Resting ECG-It shows the result of ECG & $0,1,2$ \\
\hline 8. & Heartbeat- shows the max heart beat of patient & Multiple values from 71 to 202 \\
\hline 9. & Exang- used to identify if there is an exercise induced angina. If yes $=1$ or el se no $=0$ & 0,1 \\
\hline 10. & Old Peak- describes patients depression level. & Multiple values between 0 to 6.2 . \\
\hline 11. & $\begin{array}{l}\text { Slope- describes patient condition during peak exercise. It is divided into three } \\
\text { segments(Unsloping, Flat, Down sloping) }\end{array}$ & $1,2,3$ \\
\hline 12. & CA-Result of fluoroscopy. & $0,1,2,3$ \\
\hline 13. & $\begin{array}{l}\text { Thal- test required for patient suffering from pain in chest or difficulty in breathing. } \\
\text { There are } 4 \text { kinds of values which represent Thallium test. }\end{array}$ & $0,1,2,3$ \\
\hline 14. & Target-It is the final column of the dataset. It is class or label Colum. It represents & 0,1 \\
\hline
\end{tabular}

Table.1 Selected Cleveland Heart Disease Data Set

\section{ALGORITHMS OF ML}

Communicated in absolute numbers. This field is connected with whether the patient has a heart illness. As per the analysis, the dataset focuses on recognizing presence (esteem $0,2,3,4$ ) and non-presence (esteem 0 ). If the value is 0 (missing), the value is under 4 . 


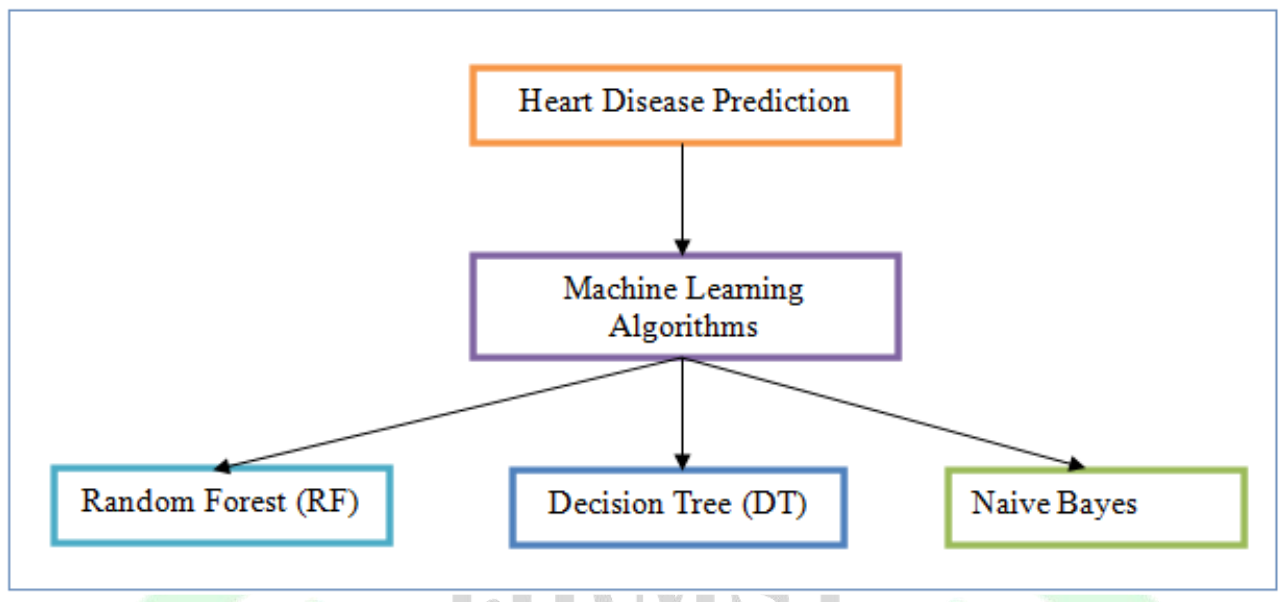

Figure 1: Prediction of Heart Disease using Machine Learning Algorithms

The following stage predicts coronary illness, which is defined in Figure 1. The correlation results show three AI calculations utilizing the coronary illness dataset, like Naive Bayes (NB), Decision Tree (DT) and Random Forest (RF) and performance models. The accompanying segments will present these three calculations.

\section{Grouping Using Random Forest}

Random Forest is the most remarkable, and it is the mix of three indicators by utilizing a decision tree. These qualities rely upon an arbitrary vector that is independently and with similar dispersion for all nodes in the random forest. This calculation consolidates arrangement and relapse dependent on the issue space. Coming up next are the means for the arbitrary random forest calculation.
- The $\mathrm{k}$ highlights are chosen randomly from general $\mathrm{m}$ elements, where $\mathrm{k} \ll \mathrm{m}$.

- Contiguous the $\mathrm{k}$ elements, compute the hub "d" utilizing the best-parted point.

- By using the best parted, the child nodes are split.

- Rehash 1 to 3 stages until 1 number of nodes has been reached.

- By rehashing 1 to 4 stages to building backwoods for $\mathrm{n}$ number occasions to make $\mathrm{n}$ number of trees.

\section{Grouping Using Decision Tree}

A Decision Tree is a short classifier to carry out. Datasets are grouped into two ways. The DT fosters the grouping or relapse model to make a's tree structure easy to handle and debug. Furthermore, DT can deal with the two sorts of 


\section{VOLUME: 12, JULY-DECEMBER 2021}

information. The data gain plays a significant part in tracking the qualities and taking out the features to divide the nodes into trees. The condition for data gain is given as:

Coming up next is Decision Tree calculation is given as:

- Observe the data acquired for the features in the dataset.

- In the deep request, the arranging is completed with the data again for the coronary illness datasets.

- After handling stage 2, the data gain is appointing the best characteristic of the dataset at the tree's foundation.

- Utilizing a similar equation, the data gain is to be determined.

- The means are to be rehashed until each character is set as child nodes in all the tree nodes.
- In light of the greatest data gain, the hubs are isolated.

\section{Using Naïve Bayes Classifier for Classification}

The naive Bayes classifier model is easy to make from complex boundaries, which makes it particularly viable in foreseeing heart sickness in the field of medication. Because of its effortlessness, the Naive Bayes classifier has colossal effects and is broadly utilized for its effectiveness better than more complex grouping procedures.

Autonomy of class conditions. A naive Bayesian classifier accepts that the value of an indicator (x) influences a given classification (c) and doesn't have anything to do with the upsides of different indicators. Bayes' hypothesis permits you to work out the back likelihood $\mathrm{P}(\mathrm{c} \mid \mathrm{x})$ from $P(c), P(x)$, and $P(x \mid c)$. 


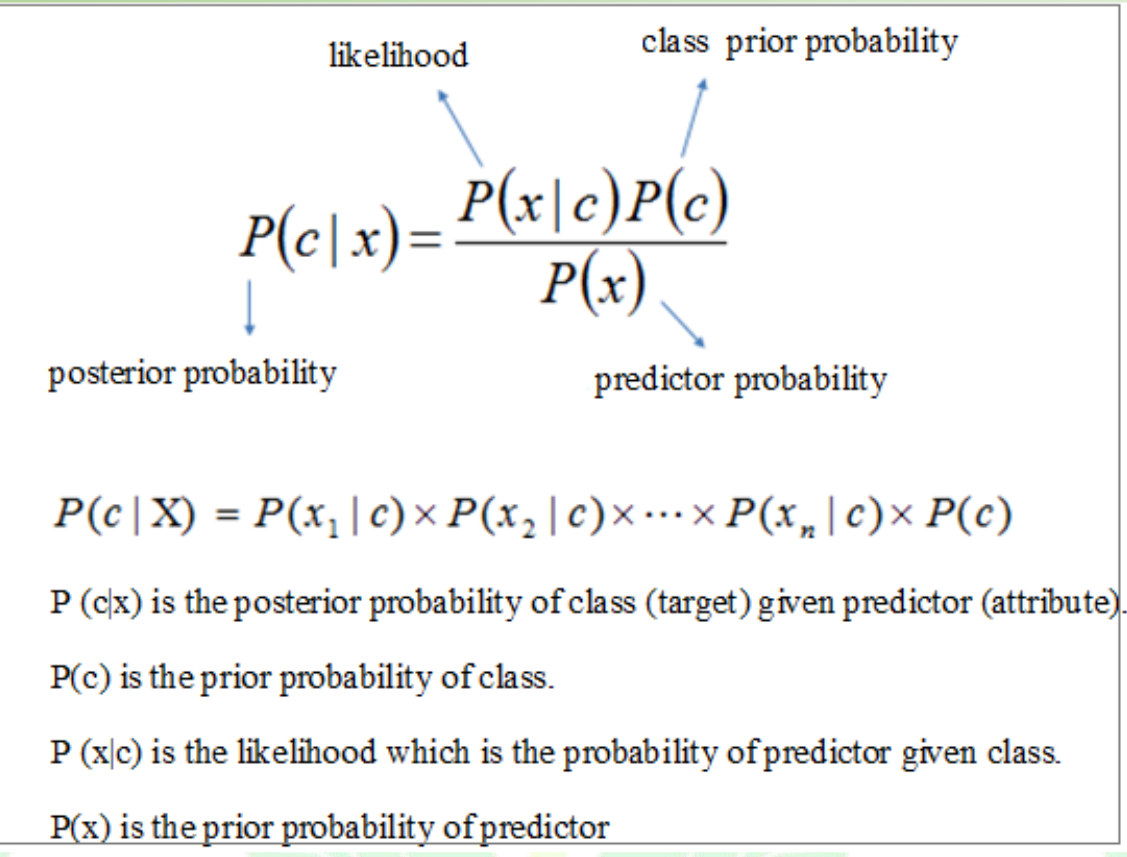

\section{An Enhanced Novel Dynamic Data Processing (ENDDP) Algorithm}

To examine the collection and mathematical information attributes as indicated by order insights. The fundamental motivation behind investigating the powerful informational collection is to get the unique data attributes of the collection. Dynamic information predominantly incorporates collected information (for example, timing information, constant information) and full unique information (for example, every one of the information embedded, removed and changed) [9]. Instalment Sensitivity is additionally one of the difficulties in special information handling. For everybody with a record, this is Global, or neighbourhood responsiveness is impacted by the weightiness of the whole informational index. An Enhanced Novel Dynamic Data Processing (ENDDP) Algorithm is an accurate classifier that doesn't suggest a disallowance between ascribes. This capacity coordinates a few Bayesian capacities for examining properties autonomous of one another. The work process of the proposed classifier is as per the following: 


\section{VOLUME: 12, JULY-DECEMBER 2021}

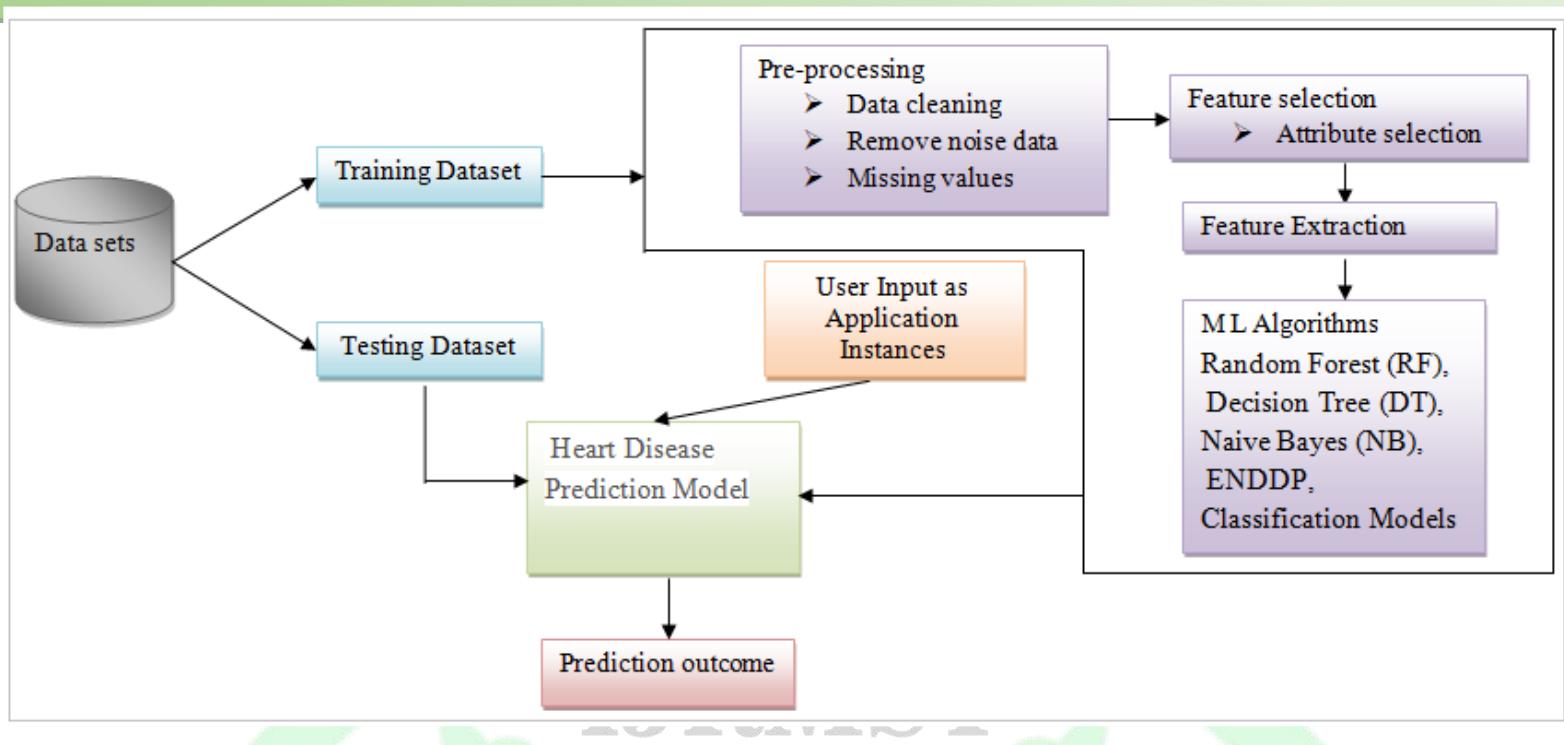

Fig 2: ENDDP working model

Accuracy: This will work out the general precision of the unusual and typical anticipated information is determined by

Accuracy $=\mathrm{TP}+\mathrm{TN} / \mathrm{TP}+\mathrm{TN}+\mathrm{FP}+\mathrm{FN}$

Execution Evolution The exhibition measures, in particular, False Positive Rate (FPR), False Negative Rate (FNR), Sensitivity, Specificity and Accuracy, the framework's presentation are assessed. The essential consider qualities such as True Positive (TP), True Negative (TN), False Positive (FP) and False Negative (FN) are utilized by these actions.

FPR ordered the level of anticipated qualities to specific and unknown information. However, it didn't.

$\mathrm{FPR}=\mathrm{FP} / \mathrm{FP}+\mathrm{TN}$

FNR The level of anticipated qualities was grouped to specific and anonymous information, yet it did.

\section{$\mathrm{FNR}=\mathrm{FN} / \mathrm{FN}+\mathrm{TN}$}

Responsiveness The up-sides are accurately recognized to work out the awareness. This is utilized to test to distinguish adverse outcomes.

Sesitivity $=\frac{\text { No. of TP }}{\text { No. of TP + No. of TN }}$ 


\section{VOLUME: 12, JULY-DECEMBER 2021}

The boundaries-based execution is displayed in table 1.

\begin{tabular}{|l|l|l|l|}
\hline $\begin{array}{l}\text { Machine } \\
\text { Learning } \\
\text { Algorithms }\end{array}$ & $\begin{array}{l}\text { Accurac } \\
\text { Naive Bayes }\end{array}$ & $\begin{array}{l}\text { Sensitivit } \\
\mathrm{y}\end{array}$ & $\begin{array}{l}\text { Specificit } \\
\mathrm{y}\end{array}$ \\
\hline Random Forest & $89.42 \%$ & $86.43 \%$ & $79.76 \%$ \\
\hline $\begin{array}{l}\text { Proposed } \\
\text { System[ENDDP } \\
\text { ] }\end{array}$ & $97.98 \%$ & $97.45 \%$ & $98.54 \%$ \\
\hline
\end{tabular}

This shows the precision of the outcome dependent on the information mining procedures.

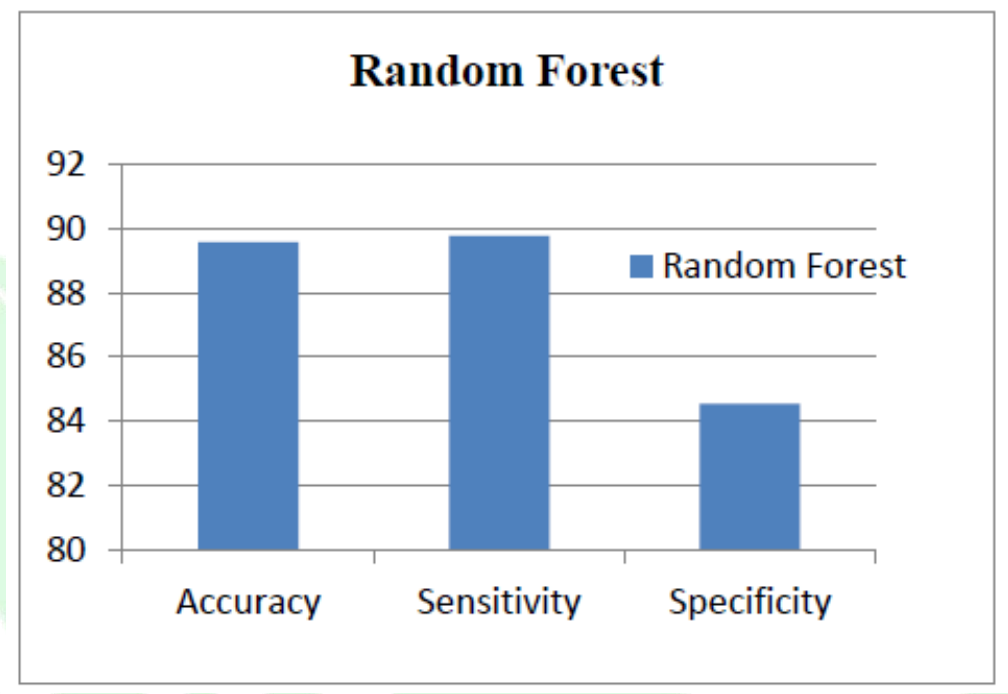

Fig 3: Random forest performance

\section{CONCLUSION}

With the expansion in passings from heart disease, the improvement of a compelling and precise coronary illness forecast framework has turned into a required assignment. This examination means tracking down the best ML algorithm to recognize coronary illness. This review analyzed the precision scores of decision trees, strategic relapse, irregular random forest, and naive Bayes calculation for foreseeing coronary illness utilizing the UCI AI archive dataset. 


\section{VOLUME: 12, JULY-DECEMBER 2021}

\section{REFERENCES}

[1]. "S. Ghwanmeh, A.Mohammad, and A.Al-Ibrahim, "Innovative artificial neural networksbased decision support system for heart diseases diagnosis," Journal of Intelligent Learning Systems and Applications, vol. 5, no. 3, pp. 176-183, 2013.

[2]. Q.K.Al-Shayea, "Artificial neural networks in medical diagnosis," International Journal of Computer Science Issues, vol. 8, no. 2, pp. 150-154, 2011.

[3]. Senthil kumar mohan, chandrasegar thirumalai and Gautam Srivastva, "Effective Heart Disease Prediction Using Hybrid Machine Learning Techniques” IEEE Access 2019.

[4]. Himanshu Sharma and M A Rizvi, "Prediction of Heart Disease using Machine Learning Algorithms: A Survey" International Journal on Recent and Innovation Trends in Computing and Communication Volume: 5 Issue: 8 , IJRITCC August 2017.

[5]. M. Nikhil Kumar, K. V. S. Koushik, K. Deepak, "Prediction of Heart Diseases Using Data Mining and Machine Learning Algorithms and Tools" International Journal of Scientific Research in Computer Science, Engineering and Information Technology ,IJSRCSEIT 2019.

[6]. Amandeep Kaur and Jyoti Arora,"Heart Diseases Prediction using Data Mining Techniques: A survey" International Journal of Advanced Research in Computer Science , IJARCS 2015-2019.

[7]. Pahulpreet Singh Kohli and Shriya Arora, "Application of Machine Learning in Diseases Prediction", 4th International Conference on Computing Communication And Automation(ICCCA), 2018.

[8]. M. Akhil, B. L. Deekshatulu, and P. Chandra, "Classification of Heart Disease Using KNearest Neighbor and Genetic Algorithm,” Procedia Technol., vol. 10, pp. 85-94, 2013.

[9]. H. Y. Kanga, *, Y. L. Mab , X. M. Sia” An Enhanced Algorithm for Dynamic Data Release Based on Differential Privacy” Procedia Computer Science 174 (2020) 15-21. 